\title{
Description of the first haemocytic rod-shaped virus from a penaeid prawn
}

\author{
Leigh Owens \\ Department of Biomedical and Tropical Veterinary Sciences, PO James Cook University of North Queensland, \\ Queensland 4811, Australia
}

\begin{abstract}
Epizootic losses of hybrid Penaeus esculentus by P. monodon crosses initiated an electron microscopical investigation. A haemocytic rod-shaped virus was found in the gill tissues as well as infectious hypodermal and haematopoietic necrosis virus in other tissues (reported elsewhere). Within the cytoplasm of small-granule haemocytes and the nucleus of other unrecognised cells, rod-shaped virions were present. The virions were $588 \times 119 \mathrm{~nm}$ with extraordinarily long virions being $888 \mathrm{~nm}$. Some were encapsulated by a smaller envelope which forced the long virions to become either $\mathrm{V}$ - or $\mathrm{U}$ shaped. Empty capsids $496 \times 50 \mathrm{~nm}$ were also seen in the nucleoplasm. Capsid originators were 50 to $60 \mathrm{~nm}$ long. The virus is named penaeid haemocytic rod-shaped virus (PHRV) until its relationship to other haemocytic viruses can be ascertained. However, PHRV showed affinities to the genus Bracovirus within the Polydnaviridae and the size and morphology were somewhat similar to rod-shaped virus of Carcinus maenus (RV-CM).
\end{abstract}

\section{INTRODUCTION}

Baculoviruses are the most commonly found viruses in crustaceans, there being at least 12 named types. Furthermore, they are often associated with disease in both penaeid prawns and portunid crabs (Johnson 1988, Johnson \& Lightner 1988). Records of the 3 haemocyte-infecting, rod-shaped viruses similar to baculoviruses are restricted at present to the portunid crabs of the Atlantic Ocean. Developmentally, they are less typically like a baculovirus and share some characteristics with polydnaviruses (Johnson 1988). During an investigation into epizootic mortalities of Penaeus esculentus hybridised with $P$. monodon, a number of viruses were visualised including infectious hypodermal and haematopoietic necrosis virus (IHHNV) and lymphoidal parvovirus (Owens et al. 1992). This paper describes the detection of a rod-shaped virus in haemocytes, which represents the first haemocytic rod-shaped virus seen in penaeid prawns.

\section{MATERIALS AND METHODS}

Hybrid prawns (Penaeus esculentus crossed with $P$. monodon) were produced and raised as described in
Owens et al. (1992). Moribund prawns were collected from holding tanks just after the major epizootic on Day 100 and fixed in Davidson's fixative. Histological processing and staining followed the methods outlined by Culling et al. (1985). For electron microscopy, diced gill tissue was fixed in $2.5 \%$ glutaldehyde $/ 2 \%$ paraformaldehyde in cacodylate buffer or $10 \%$ formalin and postfixed in $1 \%$ osmium tetroxide. The tissue was dehydrated and mounted in Spurr's (Tm) resin. Sections were cut on an LKB Ultratome at $500 \AA$ (50 nm), stained with uranyl acetate/70\% methanol and lead citrate and viewed at $80 \mathrm{kV}$ on a Jeol 2000FX transmission electron microscope. Terminology of virion components follows Federici (1986) and Johnson (1988). Measurements of virion components were taken directly from photographs or by using a cartographer's wheel for bent virions. Only intact virions which were identified by envelopes at both polar ends were used in measurements.

\section{RESULTS}

The histological findings are reported in Owens et al. (1992). Briefly, Cowdrey type A intranuclear inclusion bodies were widespread throughout all mesodermal 


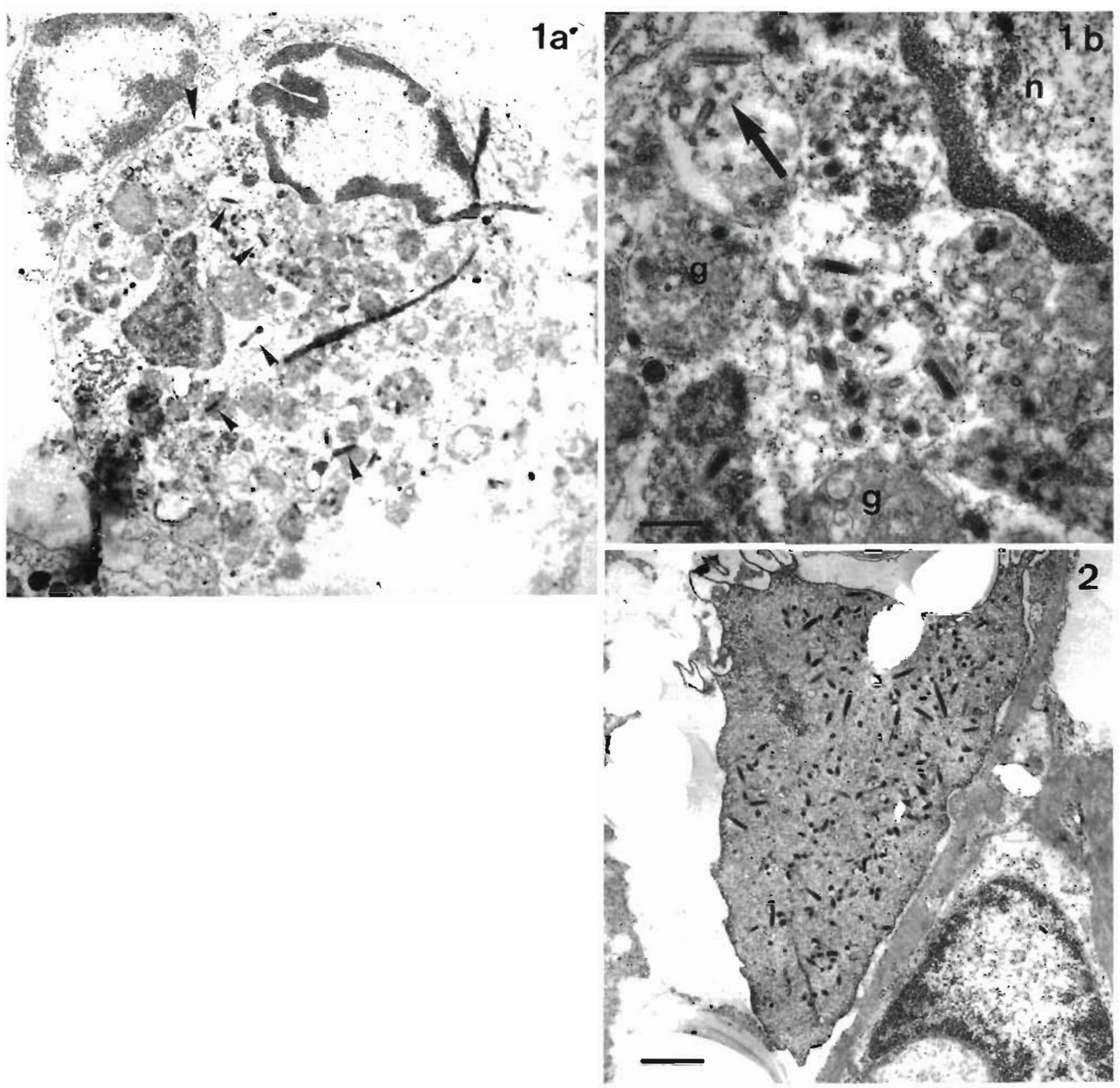

Figs. 1 to 8. Rod-shaped virus in hybrid prawns (Penaeus esculentus $\times$ P. monodon). Fig. 1a. A small-granule haemocyte showIng rod-shaped virions (small arrowheads) and empty capsids (large arrowhead) in the cytoplasm $\times 6237$. Note the rounded granules throughout the cytoplasm of the haemocyte. Scale bar $=1.6 \mu \mathrm{m}$. Fig. 1b. Higher magnification $(\times 25000)$ of Fig. 1a at the large arrowhead: small-granule haemocyte (g) with rod-shaped virions in thin-walled, cytoplasmic vacuoles. Empty capsids (arrow) and nucleocapsids are present. Note the slightly hyperchromatic nucleus $(n)$ with some nucleoplasm changes. Scale bar $=400 \mathrm{~nm}$. Fig. 2. Nucleus of an infected cell displaying dense virogenic stroma and numerous rod-shaped virions, $\times 6800$. Scale bar $=1.5 \mu \mathrm{m}$. Fig. 3. V-shaped virion within a small envelope $\times 50000$. Two enveloped, bent empty capsids are visible in cross section $(\star)$. Scale bar $=200 \mathrm{~nm}$. Fig. 4 . U-shaped virion $(\times 50000)$. Also present, a virion attached via a membrane to a capsid originator at right angles (solid arrowheads). Empty, truncated enveloped capsids are also visible (open arrowheads). Scale bar $=$ $200 \mathrm{~nm}$. Fig. 5. Cigar-shaped virions with the specialised apex visible (solid arrowheads), $\times 100000$. Some marginated chromatin is also present (c). Scale bar $=100 \mathrm{~nm}$. Fig. 6. Empty capsids (e) $\times 50000$. Two possible capsid originators (arrowheads) with 1 end still open, the one on the left clearly showing the internal originator. One possible originator attached by a membrane to a fully formed nucleocapsid. Note some cross sections of bent virions. Scale bar $=200 \mathrm{~nm}$. Fig. 7 Two capsid originators (arrowheads) $\times 25000$. Marginated chromatin is present (c) and a cytoplasmic mitochondrion $(\mathrm{m})$. Scale bar $=400 \mathrm{~nm}$. Fig 8 . Cross section of a mature virion showing the 2 protein layers of envelope (arrowheads) separated by the electron-lucent lipid laver, a slightly less electron-dense inner ring (intermediate layer, arrow) and then the nucleoprotein core, $\times 170000$. Terminology from Federici (1986). Scale bas $=53 \mathrm{~nm}$ 

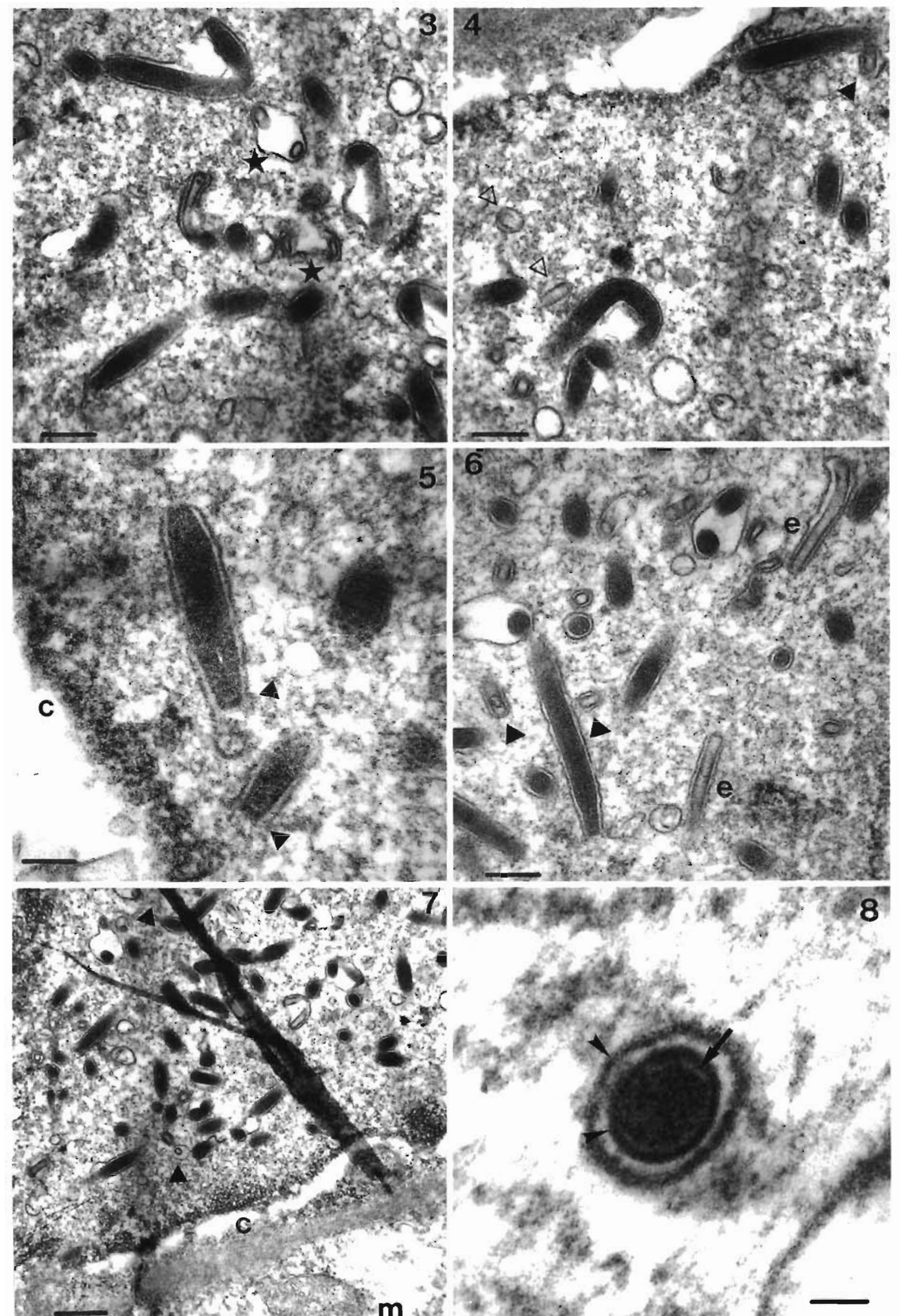
and ectodermally derived tissues. These inclusions are pathognomonic for IHHNV which is believed to be responsible for the epizootic.

In gill preparations, a number of cells were seen to harbour rod-shaped virions. The cell types included small-granule haemocytes (Fig. 1a, b) and unrecognisable cell types (Fig. 2). Bar-shaped virions, viral crosssections and empty capsids were observed within thinwalled, membrane-bound vacuoles and free in the cytoplasm of the haemocytes. The nuclei of these haemocytes displayed slight margination and darkening of the chromatin, and nucleoplasm changes but lacked virions (Fig. 1a, b). This was assumed to represent recent phagocytosis without viral replication but possible nuclear infection. Some patently infected cells showed the remnants of nuclear chromatin which identified the main area of virogenesis as the nucleus (Fig. 2). In some infected cells, the nucleus could not be distinguished nor could cytoplasmic organelles. It was assumed that the virogenic stroma had obscured organelles and that the cytoplasmic-nuclear boundaries had merged.

Many of the virions were contained singly in a envelope that caused them to bend in either a V-shape (Fig. 3) or a U-shape (Fig. 4). The extra-long, mostly bent nucleocapsids measured $888 \times 86 \mathrm{~nm}$ mean size (Table 1) whilst a second group of straight nucleocapsids were somewhat smaller at $542 \times 86 \mathrm{~nm}$ mean size. Some virions had a cigar or baseball bat shape (Fig. 5). No evidence of the nucleocapsids acquiring either a primary or secondary envelope by budding through the nuclear or cell membranes was seen. The single envelope appeared to be totally formed de novo.

Infected cells often had partially enveloped, empty capsids throughout their contents (Fig. 6). The empty capsids (presumed to be fully formed) measured $496 x$ $83 \mathrm{~nm}$ mean size including the envelope. These empty capsids were smaller than filled nucleocapsids. Many fully enveloped but empty capsids were seen and they were sometimes bent within the envelope (Fig. 3).

A number of capsid originators were seen, some in association with almost complete virions but still shar- ing a membrane (Fig. 6). The membranes did not fully enclose the capsid originators which measured 50 to $60 \mathrm{~nm}$ (Figs. 6 \& 7). The specialised apex thought to develop from the capsid originator (Johnson 1988) was not easily recognised in these preparations (Fig. 5).

Cross sections of virions showed the envelope to have 2 electron-dense protein rings separated by an electron-lucent lipid ring (Fig. 8). Inside the envelope, just preceding the capsid and nucleoprotein core, the intermediate layer (slightly electron-lucent) was visible.

\section{DISCUSSION}

The presence of empty capsids and capsid originators as well as complete virions suggests that viral replication is taking place and that the prawn is not acting only as a carrier host. As haemocytes are the only identifiable cell type that had virions present, and considering the great similarity of this virus to the crab haemocyte-infecting rod-shaped viruses, it is believed that haemocytes are a tissue that supports virogenesis of this virus. The present case represents the first record of a rod-shaped viral infection of the haemocytes of a penaeid or, indeed, any non-portunid marine crustacean. It increases the known range of haemocytic rodshaped viruses to the Pacific Ocean and Australia.

The virions, nucleocapsids and capsids are considerably longer than any other haemocytic rod-shaped virus (Table 1). Similarly, virion and nucleocapsid widths are also greater than for Rod-B. However, empty capsid width is less than either RV-CM or Rod$B$ whilst nucleocapsid width is intermediate between them (Table 1). This phenomenon of the capsid expanding when filled with nucleoprotein is common in haemocytic rod-shaped viruses (Johnson 1988). Based on morphometrics, the current haemocytic rod-shaped virus is dissimilar to known portunid haemocytic rodshaped viruses. Therefore, the virus herein is named penaeid haemocytic rod-shaped virus (PHRV) until more is known about its relationship to other haemocytic rod-shaped viruses.

Table 1. Morphometrics ( $\mathrm{nm}$ ) of penaeid haemocytic baculovirus and comparisons with other haemocytic baculoviruses

\begin{tabular}{|c|c|c|c|c|c|}
\hline & PHRV & $\mathrm{RV}-\mathrm{CM}$ & Rod-B & Bracovirus & Ichnovirus \\
\hline Extraordinary virion length & $840-920$ & $95-760$ & - & $30-150$ & - \\
\hline Ordinary virion length & $430-640$ & $370-390$ & - & - & - \\
\hline Virion width & $112-125$ & $85-100$ & - & - & - \\
\hline Nucleocapsid length & $410-600$ & $190-540$ & $220-260$ & $30-150$ & 330 \\
\hline Nucleocapsid width & $80-90$ & $95-110$ & $75-85$ & 40 & 85 \\
\hline Empty capsid length & $442-525$ & $210-280$ & $220-280$ & - & - \\
\hline Empty capsid width & $45-55$ & $65-70$ & $53-60$ & - & - \\
\hline Capsid originators & $50-60$ & $53-64$ & $53-64$ & - & - \\
\hline
\end{tabular}


Of interest was the total lack of budding of the PHRV nucleocapsid through either the nuclear membrane or the cellular membrane to acquire the envelope. In the gut-infecting baculoviruses, non-occluded virions have been shown to acquire their envelope in this manner, especially those that are going to infect other cells in the same host (Federici 1986). Similarly, nonoccluded baculoviruses of marine animals such as Scylla serrata receive their second envelope via budding (Anderson \& Prior 1992).

PHRV shows affinities to the Bracovirus within the Polydnaviridae (Francki et al. 1991). In particular, the great variable length of the nucleocapsids and the presence of only 1 de novo-produced envelope are indicative of Bracovirus. The variable length of the virions is also suggestive of segmented DNA. PHRV is also somewhat similar to the Ichnovirus within the same family in that a few smaller virions tend to be prolate ellipsoids (cigar shaped) and the size of the virions is closer to that reported for Ichnovirus than that reported for Bracovirus. However, the Ichnovirus have 2 envelopes, 1 of which is acquired by budding, not 1 de novo envelope as seen here. Johnson (1988) suggested that the rod-shaped haemocytic infecting viruses of Crustacea showed several similarities to the Polydnaviridae and this virus supports those suggestions.

The concurrent infection with IHHNV may have allowed expression of an otherwise latent virus. Alternatively, the host may possibly have been genetically immuno-compromised (Owens et al. 1992), and the penaeid may not be the normal host for this virus. Indeed, the fact that all previous cases have been in

Responsible Subject Editor: J. E. Stewart, Dartmouth, N. S., Canada portunid crabs suggests that local portunids should be examined for further cases of PHRV. Further investigations for PHRV are being undertaken so a clearer picture of its epidemiology can be obtained.

Acknowledgements. Prawns used in this study were supplied from the Australian Institute of Marine Science. Mr S. de Beer is thanked for technical help on the electron microscope. This research was funded by a Merit Research Allocation from James Cook University.

\section{LITERATURE CITED}

Anderson, I. G., Prior, H. C. (1992). Baculovirus infections in the mud crab, Scylla serrata, and a freshwater crayfish, Cherax quadricarinatus, from Australia. J. Invertebr. Pathol. 60: 265-273

Culling, C. F. A., Allison, R. T., Barr, W. T. (1985). Cellular pathology techniques,. 4 th edn. Butterworths, London

Federici, B. A. (1986). Ultrastructure of baculoviruses. In: Granados, R. R., Federici, B. A. (eds.) The biology of baculoviruses. Vol. I. Biological properties and molecular biology. CRC Press, Boca Raton, p. 61-88

Francki, R. I. B., Fauquet, C. M., Knudson, D. L., Brown, F. (1991). Classification and nomenclature of viruses. Arch. Virol. Suppl. 2: 1-450

Johnson, P. T (1988). Rod-shaped nuclear viruses of crustaceans: hemocyte-infecting species. Dis. aquat. Org. 5: $111-122$

Johnson, P. T., Lightner, D. V. (1988). Rod-shaped nuclear viruses of crustaceans: gut-infecting species. Dis. aquat. Org. 5: 123-141

Owens, L., Anderson, I. G., Kenway, M., Trott, L., Benzie, J. A. H. (1992). Infectious hypodermal and haematopoietic necrosis virus (IHHNV) in a hybrid penaeid prawn from tropical Australia. Dis. aquat. Org. 14: 219-228

Manuscript first received: December 30, 1992 Revised version accepted: May 4, 1993 